\title{
Efficacy of adjuvant radiotherapy in non-extremity soft tissue sarcoma with moderate chemosensitivity
}

\author{
Eun Mi Lee, MD1', Dong Hyun Kim, MD, PhD², Do Young Kim, MD³, \\ Young Mi Seol, MD, PhD ${ }^{3}$, Young Jin Choi, MD, PhD ${ }^{3}$, Hyojeong Kim, $\mathrm{MD}^{3}$ \\ 'Division of Hemato-oncology, Department of Internal Medicine, Kosin University College of Medicine, Busan; \\ ${ }^{2}$ Department of Radiation Oncology, Pusan National University School of Medicine, Busan; \\ ${ }^{3}$ Division of Hemato-oncology, Department of Internal Medicine, Pusan National University School of Medicine, Busan, Korea
}

Purpose: Soft tissue sarcoma (STS) is a rare and heterogeneous cancer with over 50 known subtypes. It is difficult to understand the role of adjuvant treatment in STS. We aimed to determine the benefits of adjuvant treatment for a rare STS subset: nonextremity STS with moderate chemosensitivity.

Materials and Methods: We reviewed medical records from Pusan National University Hospital and Kosin University Gospel Hospital, which had detailed pathological reports on patients diagnosed between 2006 and 2016. The most important inclusion criterion was resection with curative intent. We grouped STS by chemosensitivity based on reported data and analyzed nonextremity STS with moderate chemosensitivity.

Results: We investigated 142 patients with 20 pathological subtypes of STS. Eighty-six patients had extremity STS and 56 had nonextremity STS. Thirty-eight of 56 patients were categorized as having moderate chemosensitivity. Seventeen of 38 patients (44.7\%) received adjuvant radiotherapy and 14 (36.8\%) received adjuvant chemotherapy. A log-rank test showed longer disease-free survival (DFS) in the adjuvant radiotherapy group than in the group treated without adjuvant radiotherapy (not reached vs. 1.468 years, $p=$ 0.037). Multivariate Cox proportional hazard analysis, with covariates including age, stage, resection margin, adjuvant chemotherapy, and adjuvant radiotherapy, revealed that adjuvant radiotherapy was associated with longer DFS (odds ratio $=0.369, p=0.045$ ). Overall survival was not correlated with adjuvant radiotherapy.

Conclusion: Adjuvant radiotherapy may be associated with longer DFS in patients with non-extremity STS with moderate chemosensitivity.

Keywords: Adjuvant chemotherapy, Adjuvant radiotherapy, Soft tissue sarcoma, Survival

\section{Introduction}

Sarcoma is a malignant cancer of skeletal and extraskeletal connective tissue. Soft tissue sarcoma (STS) is a rare form of tumor which represents 1\% of all malignancies [1]. One of the main challenges in investigating rare diseases like STS is the lack of systemic data and clinical evidence. STS has more than 50 pathological subtypes [2] that can occur at all anatomic sites although some subtypes preferentially develop at specific anatomic locations. The National Comprehensive Cancer

Received 31 May 2018, Revised 25 September 2018, Accepted 24 October 2018.

Correspondence: Hyojeong Kim, MD, Division of Hemato-oncology, Department of Internal Medicine, Pusan National University School of Medicine, 179 Gudeok-ro, Seo-gu, Busan 49241, Korea. Tel: +82-51-240-7532, Fax: +82-51-256-8330, E-mail: leonkim80@pusan.ac.kr (https://orcid.org/0000-0002-0943-5241)

(c) This is an Open Access article distributed under the terms of the Creative Commons Attribution Non-Commercial License (http://creativecommons.org/ licenses/by-nc/4.0/) which permits unrestricted non-commercial use, distribution, and reproduction in any medium, provided the original work is properly cited.

www.e-roj.org 
Network Guidelines recommend multiple options for patients with various subtypes of STS [3]. However, there is no gold standard treatment for STS, as the disease presentation can be quite diverse.

Similar to treatment for most solid cancers, complete surgical resection is a prerequisite for curing STS. Furthermore, adjuvant treatment following surgical resection can improve patient survival. Currently, not enough data and evidence exist on the different subtypes of STS. However, it is known that most STS occurs in the extremities [4]. Clinical information about non-extremity STS is even more limited. Therefore, treatment decisions for non-extremity STS are sometimes made by extrapolating from extremity STS. However, nonextremity STS behavior different from extremity STS and it is more difficult to achieve enough surgical margin in nonextremity STS. We conducted a retrospective analysis of non-extremity STS survivors to identify the role of adjuvant treatment. We grouped STS by chemosensitivity based on a guideline and reported data, for reducing heterogeneity with extreme diversity of STS. Adjuvant radiotherapy (RT) has substantial evidence than adjuvant chemotherapy in extremity STS. We aimed to investigate the efficacy of adjuvant RT or adjuvant chemotherapy in non-extremity STS with moderate chemosensitivity.

\section{Materials and Methods}

\section{Patients}

We reviewed the medical records of patients who were diagnosed with STS at Pusan National University Hospital and Kosin University Gospel Hospital between January 2006 and December 2016. The inclusion criteria were as follows: (1) those over the age of 18 years, (2) those who underwent surgical resection with curative intent, (3) patients with nonextremity tumors and (4) those with tumors with moderate chemosensitivity. A total of 142 patients with 20 pathological subtypes of STS underwent surgery with curative intent. Two patients with STS in chest wall and perirenal area and 1 patient with STS in right thigh were excluded because they had $\mathrm{R} 2$ resection margin indicating a remnant tumor. Failure to achieve complete resection was defined as tumor microscopically within or less than $1 \mathrm{~mm}$ from the resection margin [5-8]. Microscopically incomplete resection was classified as R1, while complete resection was classified as Ro. The adjuvant treatment is generally indicated with R0 resection. The indication widely includes $R 1$ resections. The $R 2$ resections indicate curative or palliative treatments.
The UK guidelines for managing STS in 2016 report on the relative chemosensitivity of various STS subtypes [9]. They grouped STS into five categories according to chemosensitivity: (1) chemotherapy integral to management, (2) chemosensitive, (3) moderately chemosensitive, (4) relatively chemoinsensitive, and (5) chemo-insensitive. The Institutional Review Board of the Pusan National University Hospital approved this retrospective study (No. H-1803-005-064).

\section{Statistics}

Our primary objective was to investigate the efficacy of adjuvant RT or chemotherapy in patients with non-extremity STS. Efficacy measures included disease-free survival (DFS) and overall survival (OS). OS was defined as the time between surgical resection and death from any cause. DFS was defined as the time between surgical resection and disease recurrence or death due to any cause. Survival was estimated by the Kaplan-Meier method and compared by a log-rank test. A multivariate Cox proportional hazards regression model was used to identify prognostic or predictive factors. Survival analyses were performed using SPSS Statistics version 22 (IBM, Armonk, NY, USA), and significance was defined as a twotailed of $\leq 0.05$.

\section{Results}

\section{Patient characteristics}

We investigated 142 patients with 20 pathological subtypes of STS, grouped into three categories according to chemosensitivity (Table 1). Eighty-six patients (61\%) had extremity STS and 56 (39\%) had non-extremity STS. Eleven of 56 patients had pathological subtypes which were regarded as sensitive to chemotherapy. Those were alveolar rhabdomyosarcoma (1 patient), Ewing sarcoma (1), uterine leiomyosarcoma (3), myxoid liposarcoma (3), primitive neuroectodermal tumor (1), and synovial sarcoma (2). Thirty-eight of 56 patients had moderate chemosensitivity, and were grouped as follows: angiosarcoma, non-uterine leiomyosarcoma, myxofibrosarcoma, malignant peripheral nerve sheath tumor, undifferentiated pleomorphic sarcoma dedifferentiated liposarcoma, well-differentiated liposarcoma epithelioid sarcoma, and intimal sarcoma. Seven of 56 patients had non-extremity STS with insensitivity to chemotherapy. They included 5 myxofibrosarcomas, 1 alveolar soft part sarcoma, and 1 dermatofibrosarcoma protuberance.

These 38 patients with non-extremity STS with moderate chemosensitivity were analyzed. Undifferentiated pleomorphic 
Table 1. STS grouped by chemosensitivity

\begin{tabular}{|c|c|}
\hline $\begin{array}{c}\text { Relative } \\
\text { chemosensitivity }\end{array}$ & Examples of STS \\
\hline Chemosensitive & $\begin{array}{l}\text { Ewing sarcoma family tumors } \\
\text { Alveolar rhabdomyosarcoma } \\
\text { Synovial sarcoma } \\
\text { Myxoid/round cell liposarcoma } \\
\text { Uterine leiomyosarcoma }\end{array}$ \\
\hline $\begin{array}{l}\text { Moderately che- } \\
\text { mosensitive }\end{array}$ & $\begin{array}{l}\text { Angiosarcoma } \\
\text { Non-uterine leiomyosarcoma } \\
\text { Myxofibrosarcoma } \\
\text { Malignant peripheral nerve sheath tumor } \\
\text { Undifferentiated pleomorphic sarcoma } \\
\text { Dedifferentiated liposarcoma } \\
\text { Well-differentiated liposarcoma } \\
\text { Pleomorphic liposarcoma } \\
\text { Epithelioid sarcoma } \\
\text { Intimal sarcoma } \\
\text { Fibrosarcoma } \\
\text { Rhabdomyosarcoma, not otherwise specified } \\
\text { Liposarcoma, not otherwise specified }\end{array}$ \\
\hline $\begin{array}{l}\text { Chemo-insensi- } \\
\text { tive }\end{array}$ & $\begin{array}{l}\text { Dermatofibrosarcoma protuberans } \\
\text { Alveolar soft part sarcoma }\end{array}$ \\
\hline
\end{tabular}

STS, soft tissue sarcoma.

sarcoma (9 patients) was the most common subtype, followed by non-uterine leiomyosarcoma. Epithelioid sarcoma and intimal sarcoma were diagnosed in one patient each. The anatomical locations were variable (Table 2). The median follow-up time was 39.5 months.

\section{Adjuvant treatment and survival}

Seventeen of the 38 patients (44.7\%) received adjuvant RT and $14(36.8 \%)$ were treated with adjuvant chemotherapy. Three of 14 patients with $\mathrm{R} 1$ resection margins were treated with adjuvant RT followed by chemotherapy. The median DFS of all patients was 3.9 years, and the median OS had not yet been reached. Table 3 shows that there was no significant difference in clinical characteristics between the patients treated with and without adjuvant RT.

Radiation was delivered using external beam radiation therapy (EBRT) alone with a 6- or 10-MV photons to all patients. Intensity-modulated RT and 3D conformal RT were used in 10 and 7 patients, respectively. After 44-50 Gy irradiation to planning target volume 1 (PTV1), the boost dose of 6-16 Gy with a shrinking field technique was delivered to PTV2. The definition of clinical target volume 1 (CTV1) was tumor bed with 3-4 cm craniocaudal and $1.5 \mathrm{~cm}$ radial expansion. The CTV2 was defined as tumor bed with $1.5 \mathrm{~cm}$ margin. The median PTV margin was $0.5 \mathrm{~cm}$ (range, 0.5 to 1.2 $\mathrm{cm})$. A log rank test showed that adjuvant RT was correlated with longer DFS in non-extremity STS with moderate chemosensitivity (not reached vs. 1.468 years, $p=0.037$ ) (Fig. 1). There was no significant correlation between adjuvant RT and OS (6.049 years vs. not reached, $p=0.941)$. Clear resection margins and adjuvant chemotherapy were not significantly correlated with DFS or OS. Similarly, adjuvant treatment following surgical resection had no effect on survival.

Multivariate Cox proportional hazards analysis, with covariates including age, stage, resection margin, adjuvant chemotherapy, and adjuvant RT, revealed that adjuvant RT was associated with longer DFS (odds ratio $=0.369, p=0.045$ ). Having stage III cancer was also correlated with shorter DFS (odds ratio $=0.256, p=0.051$ ). However, OS showed no correlation with adjuvant RT or adjuvant chemotherapy.

\section{Discussion and Conclusion}

STS is one of the most challenging tumors to treat because of its heterogeneity and lack of solid clinical evidence on effective treatment and management. However, we could group tumors into more homogenous groups according to chemosensitivity. Chemosensitivity was estimated using the 2016 UK guidelines for the management of STS and available literatures [912]. Angiosarcoma and non-uterine leiomyosarcoma are considered to have moderate chemosensitivity according

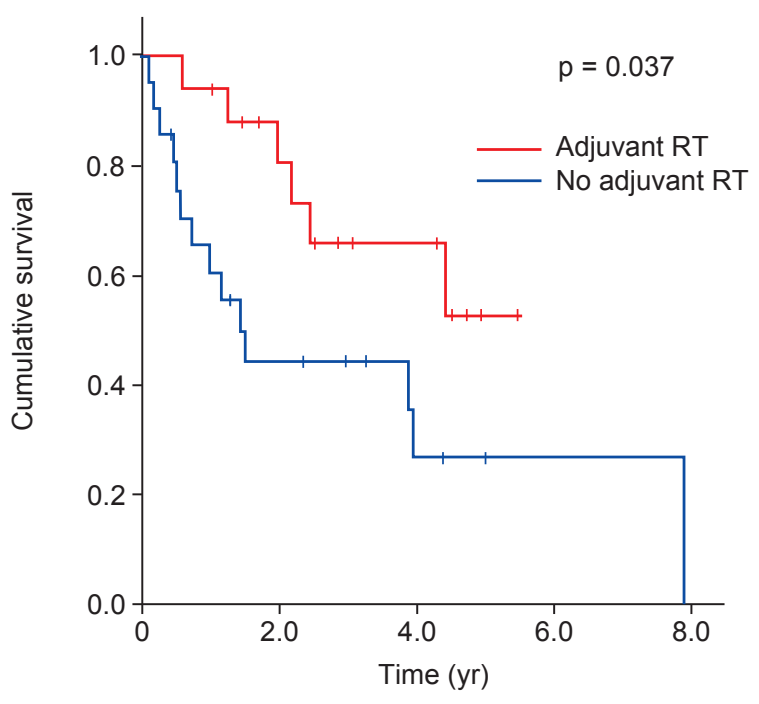

Fig. 1. Disease-free survival (DFS) curves for non-extremity soft tissue sarcoma with moderate chemosensitivity show higher DFS rates with adjuvant radiotherapy (RT). 
Table 2. Pathological and anatomical characteristics of patients with non-extremity STS with moderate chemosensitivity

\begin{tabular}{|c|c|c|c|}
\hline Pathology & Anatomical location & $\begin{array}{l}\text { Total no. } \\
\text { of patients }\end{array}$ & Adjuvant treatment \\
\hline Epithelioid sarcoma & $\operatorname{Rib}(1)$ & 1 & RT \\
\hline Intimal sarcoma & Pulmonary artery (1) & 1 & Chemotherapy \\
\hline Angiosarcoma & Scalp (1) & 2 & No \\
\hline Well differentiated liposarcoma & $\begin{array}{l}\text { Retroperitoneum (1) } \\
\text { Scrotum (1) }\end{array}$ & 2 & No \\
\hline Malignant peripheral nerve sheath tumor & $\begin{array}{l}\text { Back (2) } \\
\text { Prostate (1) } \\
\text { Retroperitoneum (1) }\end{array}$ & 4 & RT in 2 of back \\
\hline Myxofibrosarcoma & $\begin{array}{l}\text { Abdomen wall (1) } \\
\text { Chest wall (2) } \\
\text { Pelvis (1) } \\
\text { Retroperitoneum (1) }\end{array}$ & 5 & $\begin{array}{l}\text { RT in pelvis, retroperitoneum, and } 2 \text { of } \\
\text { chest wall }\end{array}$ \\
\hline Dedifferentiated liposarcoma & $\begin{array}{l}\text { Abdomen wall (1) } \\
\text { Retroperitoneum (3) } \\
\text { Inguinal area (1) } \\
\text { Neck (1) }\end{array}$ & 6 & $\begin{array}{l}\text { Chemotherapy in inguinal area and } 2 \text { of } \\
\text { retroperitoneum }\end{array}$ \\
\hline Leiomyosarcoma & $\begin{array}{l}\text { Abdomen wall (1) } \\
\text { Back (1) } \\
\text { Chest wall (1) } \\
\text { Cecum (1) } \\
\text { Mesentery (1) } \\
\text { Penis (2) } \\
\text { Retroperitoneum (1) }\end{array}$ & 8 & $\begin{array}{l}\text { Chemotherapy in back, cecum, mesentery, } \\
\text { retroperitoneum, and } 2 \text { of penis } \\
\text { RT and chemotherapy in abdominal wall }\end{array}$ \\
\hline Undifferentiated pleomorphic sarcoma & $\begin{array}{l}\text { Abdomen wall (3) } \\
\text { Back (1) } \\
\text { Breast (1) } \\
\text { Chest wall (1) } \\
\text { Duodenum (1) } \\
\text { Kidney (1) } \\
\text { Larynx (1) }\end{array}$ & 9 & $\begin{array}{l}\text { RT in back, larynx, and } 2 \text { of abdominal } \\
\text { wall } \\
\text { Chemotherapy in breast, chest wall and } \\
\text { duodenum } \\
\text { RT and chemotherapy in } 1 \text { abdominal wall }\end{array}$ \\
\hline
\end{tabular}

STS, soft tissue sarcoma; RT, radiotherapy.

to the guideline. And malignant peripheral nerve sheath tumors, myxofibrosarcoma, and dedifferentiated liposarcomas are regarded as relatively chemo-insensitive tumors. Undifferentiated pleomorphic sarcoma (UPS), which was previously called malignant fibrous histiocytoma, is not described in the guidelines. UPS reportedly responds to gemcitabine, although its chemosensitivity has not been clarified [10]. Well-differentiated liposarcoma is considered to have similar chemosensitivity to dedifferentiated liposarcoma $[11,12]$.

Complete surgical resection is the most important treatment for curing most STS. In addition, nomograms have been developed incorporating patient age, tumor size, tumor grade, tumor depth, and tumor histology to predict postoperative survival $[13,14]$. In this study, we regarded as that T stage of staging include tumor size. Surgical resection in combination with RT has achieved local control rates exceeding 90\% for extremity STS and approximately 60\% for retroperitoneal sarcoma [15]. Two randomized trials also demonstrated that adjuvant RT following limb-sparing surgery reduced local recurrence $[16,17]$. A report based on the Surveillance, Epidemiology, and End Results (SEER) data suggested that there was an OS benefit from adjuvant radiotherapy in stage III extremity sarcoma [18]. Although there have been no randomized clinical trials, retrospective and case-control studies have demonstrated that adjuvant RT, compared with surgery alone, reduces the risk of local recurrence and prolongs the recurrence-free survival of patients with retroperitoneal STS $[19,20]$.

These favorable results from trials in extremity STS and 
Table 3. Clinical characteristics of patients with non-extremity STS with moderate chemosensitivity $(n=38)$

\begin{tabular}{|c|c|c|c|c|}
\hline Characteristic & Adjuvant RT & & No adjuvant RT & $p$-value \\
\hline No. of patients & $17(44.7)$ & & $21(55.3)$ & \\
\hline DFS (yr) & Not reached & & 1.468 & 0.037 \\
\hline Median & & 3.9 & & \\
\hline OS (yr) (median) & 6.0 & & Not reached & 0.941 \\
\hline Median & & Not reached & & \\
\hline 3 -yr survival rate $(\%)$ & 47 & & 61 & 0.036 \\
\hline Median & & 55 & & \\
\hline \multicolumn{5}{|l|}{ Age (yr) } \\
\hline$<55$ & 5 & & 11 & \\
\hline$\geq 55$ & 11 & & 9 & 0.154 \\
\hline \multicolumn{5}{|l|}{ Sex } \\
\hline Female & 4 & & 10 & \\
\hline Male & 12 & & 10 & 0.126 \\
\hline \multicolumn{5}{|l|}{ Stage } \\
\hline I, II & 12 & & 18 & \\
\hline III & 4 & & 2 & 0.374 \\
\hline \multicolumn{5}{|l|}{ Complete resection } \\
\hline Achieved & 4 & & 9 & \\
\hline Failed & 12 & & 11 & 0.301 \\
\hline \multicolumn{5}{|l|}{ Adjuvant chemotherapy } \\
\hline Yes & 3 & & 10 & \\
\hline No & 13 & & 10 & 0.083 \\
\hline
\end{tabular}

STS, soft tissue sarcoma; RT, radiotherapy; DFS, disease-free survival; OS, overall survival.

Analyses were performed with Fisher exact, $\chi^{2}$, and log-rank tests.

reports in retroperitoneal STS could support adjuvant RT in non-extremity STS. However, there is still uncertainty in definite benefit of adjuvant RT in non-extremity STS after surgical resection achieving $\mathrm{RO}$ or R1 margin. In this study, there were 11 patients who did not receive adjuvant RT even though they had R1 margins. Seven of those 11 patients received adjuvant chemotherapy but, 4 of them had no adjuvant treatment. Two patients who were 20 and 21 years old had 2.7 and 1.3 months of PFS. We assume that the uncertainty or delay of precise diagnosis of STS in their retroperitoneum and prostate could affect the decisions. And 73-year-old and 76-year-old patients had some comorbidities. Also we assume that the doctors could not be sure that the benefit of adjuvant treatment would exceed the risk of injury from it in this disease entity.

There are substantial concerns about radiation-induced visceral injury in non-extremity STS unlikely in extremity STS. The gastrointestinal epithelium is susceptible to injury by RT or chemotherapy because it has a high proliferative rate. Radiation cause mucosal stem cell damage and leukocytes infiltration. These are followed by microvascular and lymphatic damage, crypt abscess formation, mucosal edema and ischemia. RT on abdomen can acutely induce nausea, vomiting, anorexia, diarrhea and abdominal pain from gastritis or enteritis. Late effects include malabsorption and ulceration. RT on chest can typically induce lung injury. RT-induced lung injury can occur by direct cytotoxicity and followed fibrosis of lung tissue. Symptoms caused by acute radiation pneumonitis usually develop within 3 months following irradiation, whereas symptoms of late or fibrotic radiation pneumonitis develop after 6 to 12 months. They include a nonproductive cough, dyspnea, low-grade fever, chest pain and malaise. RT on chest can cause dysphagia and odynophagia from esophagitis as well. It can also induce cardiac toxicity leading to heart failure, pericarditis or arrhythmia. Newer highly focused radiation techniques, such as intensity-modulated radiotherapy and charged particles, will probably play an increasing role in the management of STS in anatomically critical locations [21]

Adjuvant chemotherapy is not a standard treatment for STS, but it could be an option for high-risk patients with uncertain conditions [22]. Despite many randomized trials, the available evidence for the role of adjuvant chemotherapy is conflicting. Certain tumor types, like Ewing sarcoma and embryonal and alveolar rhabdomyosarcoma, are very 
chemosensitive, and therefore chemotherapy is integral to their management. We assumed that adjuvant chemotherapy would improve the survival of patients with chemosensitive STS. Further investigations are needed to clarify how adjuvant chemotherapy can contribute to improving survival in some subgroups.

The adjuvant RT in extremity STS has shown some survival benefit although there is no definite data from randomized clinical trial. However, there is very limited data about adjuvant treatment following surgical resection in non-extremity STS. And RT on non-extremity locations have more concerns about RT-induced visceral injury. Therefore, we cannot help treating the patients with non-extremity STS case by case amid considerable uncertainty. In this study, adjuvant RT, unlike chemotherapy, was correlated with longer DFS in nonextremity STS with moderate chemosensitivity. However, adjuvant RT did not improve OS. This might be because of the limitations of this study, like its small number of patients, the relatively short follow-up time for some patients, and its retrospective nature. To overcome these limitations, we need more systemic data. Further, the number of STS cases in each subtype was too small to analyze for statistical significance.

In conclusion, the adjuvant RT following surgical resection in non-extremity STS have problems like very limited data of evidence and risk of visceral injury. This study suggest that the adjuvant RT may be associated with longer DFS in patients with non-extremity STS with moderate chemosensitivity.

\section{Conflict of Interest}

No potential conflict of interest relevant to this article was reported.

\section{Acknowledgments}

This work was supported by clinical research grant from Pusan National University Hospital in 2018.

\section{References}

1. Siegel $R$, Naishadham $D$, Jemal A. Cancer statistics, 2013. CA Cancer J Clin 2013;63:11-30.

2. Wang J, Zhu XZ. Introduction of WHO classification of tumours of soft tissue, the fourth edition. Zhonghua Bing Li Xue Za Zhi 2013;42:363-5.

3. von Mehren $M$, Randall $R L$, Benjamin RS, et al. Soft Tissue Sarcoma, Version 2.2016, NCCN Clinical Practice Guidelines in
Oncology. J Natl Compr Canc Netw 2016;14:758-86.

4. Lawrence $W$ Jr, Donegan WL, Natarajan N, Mettlin C, Beart $R$, Winchester D. Adult soft tissue sarcomas: a pattern of care survey of the American College of Surgeons. Ann Surg 1987;205:349-59.

5. Eilber FC, Rosen G, Nelson SD, et al. High-grade extremity soft tissue sarcomas: factors predictive of local recurrence and its effect on morbidity and mortality. Ann Surg 2003;237:218-26.

6. Weitz J, Antonescu CR, Brennan MF. Localized extremity soft tissue sarcoma: improved knowledge with unchanged survival over time. J Clin Oncol 2003;21:2719-25.

7. Gronchi A, Casali PG, Mariani L, et al. Status of surgical margins and prognosis in adult soft tissue sarcomas of the extremities: a series of patients treated at a single institution. J Clin Oncol 2005;23:96-104.

8. Potter BK, Hwang PF, Forsberg JA, et al. Impact of margin status and local recurrence on soft-tissue sarcoma outcomes. J Bone Joint Surg Am 2013;95:e151.

9. Dangoor A, Seddon B, Gerrand C, Grimer R, Whelan J, Judson I. UK guidelines for the management of soft tissue sarcomas. Clin Sarcoma Res 2016;6:20.

10. Maki RG, Wathen JK, Patel SR, et al. Randomized phase II study of gemcitabine and docetaxel compared with gemcitabine alone in patients with metastatic soft tissue sarcomas: results of sarcoma alliance for research through collaboration study 002 [corrected]. J Clin Oncol 2007;25:2755-63.

11. Italiano A, Toulmonde M, Cioffi A, et al. Advanced welldifferentiated/dedifferentiated liposarcomas: role of chemotherapy and survival. Ann Oncol 2012;23:1601-7.

12. Jones RL, Fisher C, AI-Muderis O, Judson IR. Differential sensitivity of liposarcoma subtypes to chemotherapy. Eur J Cancer 2005;41:2853-60.

13. Kattan MW, Leung DH, Brennan MF. Postoperative nomogram for 12-year sarcoma-specific death. J Clin Oncol 2002;20:7916.

14. Callegaro D, Miceli R, Bonvalot $S$, et al. Development and external validation of two nomograms to predict overall survival and occurrence of distant metastases in adults after surgical resection of localised soft-tissue sarcomas of the extremities: a retrospective analysis. Lancet Oncol 2016;17:671-80.

15. Dickie $\mathrm{Cl}$, Haas $\mathrm{R}$, O'Sullivan B. Adjuvant radiation for soft tissue sarcomas. Am Soc Clin Oncol Educ Book 2015:e634-42.

16. Pisters PW, Harrison LB, Leung DH, Woodruff JM, Casper ES, Brennan MF. Long-term results of a prospective randomized trial of adjuvant brachytherapy in soft tissue sarcoma. J Clin Oncol 1996;14:859-68. 
17. Yang JC, Chang $A E$, Baker $A R$, et al. Randomized prospective study of the benefit of adjuvant radiation therapy in the treatment of soft tissue sarcomas of the extremity. J Clin Oncol 1998;16:197-203.

18. Kachare SD, Brinkley J, Vohra NA, Zervos EE, Wong JH, Fitzgerald TL. Radiotherapy associated with improved survival for high-grade sarcoma of the extremity. J Surg Oncol 2015;112:338-43.

19. Stoeckle $E_{1}$ Coindre JM, Bonvalot $S$, et al. Prognostic factors in retroperitoneal sarcoma: a multivariate analysis of a series of 165 patients of the French Cancer Center Federation Sarcoma
Group. Cancer 2001;92:359-68.

20. Nussbaum DP, Rushing CN, Lane WO, et al. Preoperative or postoperative radiotherapy versus surgery alone for retroperitoneal sarcoma: a case-control, propensity scorematched analysis of a nationwide clinical oncology database. Lancet Oncol 2016;17:966-75.

21. Ford SJ, Almond LM, Gronchi A. An update on non-extremity soft tissue sarcomas. Clin Oncol (R Coll Radiol) 2017;29:51627.

22. Casali PG. Adjuvant chemotherapy for soft tissue sarcoma. Am Soc Clin Oncol Educ Book 2015:e629-33. 